\title{
Photobiomodulation therapy and endodontic treatment of teeth with apical periodontitis using 940-nm diode laser. Report of two cases
}

\author{
Francisco Rubio ${ }^{1}$, Federico Wienecke ${ }^{1}$, Josep Arnabat-Domínguez ${ }^{2}$, Pablo Betancourt ${ }^{3,4}$ \\ ${ }^{1}$ Faculty of Dentistry, Universidad Andrés Bello, Concepción, Chile \\ ${ }^{2}$ Department of Dentistry. Faculty of Medicine, University of Barcelona, Barcelona, Spain \\ ${ }^{3}$ Endodontic Laboratory, Center for Research in Dental Sciences (CICO), Faculty of Dentistry, Universidad de La Frontera, Te- \\ muco, Chile \\ ${ }^{4}$ Department of Integral Adultos; Faculty of Dentistry, Universidad de La Frontera, Temuco, Chile
}

Correspondence:

Endodontic laboratory

Faculty of Dentistry

Universidad de La Frontera

Avenida Francisco Salazar 01145

Teтuco, Chile

pablo.betancourt@ufrontera.cl

Rubio F, Wienecke F, Arnabat-Domínguez J, Betancourt P. Photobiomodulation therapy and endodontic treatment of teeth with apical periodontitis using 940-nm diode laser. Report of two cases. J Clin Exp Dent. 2022;14(3):e298-302.

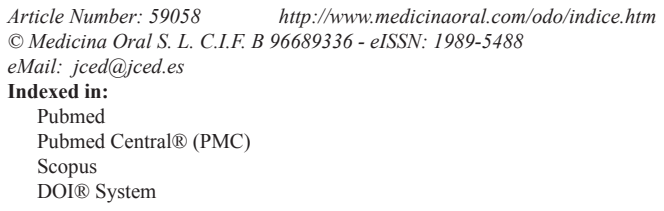

\begin{abstract}
Background: Diode laser (DL) can be used in endodontics both for its bactericidal effect inside the root canal system (RCS) and for photobiomodulation therapy (PBMT) to accelerate the repair of periradicular bone tissue.

Clinical Cases: This work presents two cases of pulp necrosis/asymptomatic apical periodontitis (AAP) that were treated with 940-nm DL, administered both to disinfect the root canal and to apply PBMT to the periradicular tissues. The cases were analysed by Cone-Beam Computed Tomography (CBCT).

Discussion: DL has become widely accepted due to its high antimicrobial effectiveness and its ability to accelerate the repair of large apical lesions by biostimulation. Nevertheless, differences of opinion persist within the scientific community due to the lack of standardized endodontic protocols.

Conclusions: The application of 940-nm DL, both for disinfection of the RCS and for PBMT, is an effective treatment in non-vital teeth with large periapical lesions. In both cases reported, bone neoformation were found at the 6-month check-up.
\end{abstract}

Key words: Low-level laser therapy, photobiomodulation therapy, diode laser, endodontics.

\section{Introduction}

Apical periodontitis (AP) is an infectious inflammatory disease, prevalent all over the world, which increases with age (1). It occurs mainly as the result of infection of the root canal system (RCS), characterised by bone in- flammation and destruction of the periradicular tissues, and may lead to tooth loss (2). The main goal in endodontics is the eradication of the microorganisms present in the whole RCS. This is extremely difficult, however, principally due to the morphological complexity of the 
tooth. Conventional treatments have proved insufficient to bring endodontic pathogenic microorganisms below detection limits, with success rates of no more than $62 \%$ to $83 \%$ in solving secondary/persistent infections (3). Thus new strategies are needed to control the bacterial biofilm and treat endodontic infections. In recent decades, interest in the use of diode laser (DL) to treat RCS has grown significantly, thanks to its affordability and broad spectrum of indications. Different studies have found that the energy released by DL in the root canal can exert a variety of effects, including photothermal disinfection and biostimulation of the periradicular bone tissue $(4,5)$.

Photobiomodulation therapy (PBMT) applied with Low-Level Laser (LLLT) has shown good results recently in accelerating wound and apical cicatrization $(6,7)$. The aim of this article was to report two cases of teeth with asymptomatic apical periodontitis (AAP) treated with PBMT and disinfection protocol by $940-\mathrm{nm}$ DL.

\section{Case Report}

Case report 1. Male patient, 21 years old, with no relevant medical history. Panoramic and periapical X-ray examination showed an osteolytic lesion with mesio-distal width of $13.2 \mathrm{~mm}$, in the area of teeth 3.2-3.1-4.1-4.2, causing slight thinning of the lingual cortical plate and expansion and fenestration of the buccal plate (Fig. 1).
Clinical examination showed a crown discoloration of tooth 3.1, soft tissues normal, percussion test and sensitivity thermal tests (ethyl chloride and guta-percha) negative. The diagnosis was pulp necrosis/AAP. Endodontic treatment of tooth 3.1 (single root canal) was carried out in 2 sessions. In the first session root canal instrumentation was carried out with a mechanised system \#40/0.4 (Reciproc, VDW, Germany). After the use of each instrument, the root canals were irrigated with 1 $\mathrm{mL}$ of $2.5 \% \mathrm{NaOCl}$ using a syringe and a 30 -gauge side-vented needle (Becton Dickinson, Madrid, Spain) to the WL. The canals were irrigated with $1 \mathrm{~mL}$ ethylenediaminetetraacetic acid 18\% (EDTA) (Ultradent, USA) for $1 \mathrm{~min}$, followed by $1 \mathrm{~mL}$ of $2.5 \% \mathrm{NaOCl}$ and $1 \mathrm{~mL}$ of saline. The irrigation protocol ended with the ultrasonic activation (Ultra X, Eighteeth, China) of $\mathrm{NaOCl} 5 \%$ for $60 \mathrm{sec}$, positioning the tip $2 \mathrm{~mm}$ short to the working length.

Dry intracanal disinfection was then performed with DL (940-nm) (EpicX, Biolase, USA), using $200 \mu \mathrm{m}$ tips and power $1.0 \mathrm{~W}$. The tip was positioned $-1 \mathrm{~mm}$ from the work length, irradiating the root canal with an apico-coronal spiral movement at $1 \mathrm{~mm}$ per sec, with 4 repetitions (10 sec between applications) (Fig. 2). Once dry, the canal was treated with calcium hydroxide (Ultracal XS, Ultradent, USA). PBMT was then applied with DL (940-nm) (EpicX, Biolase, USA), with one spot of 0.2

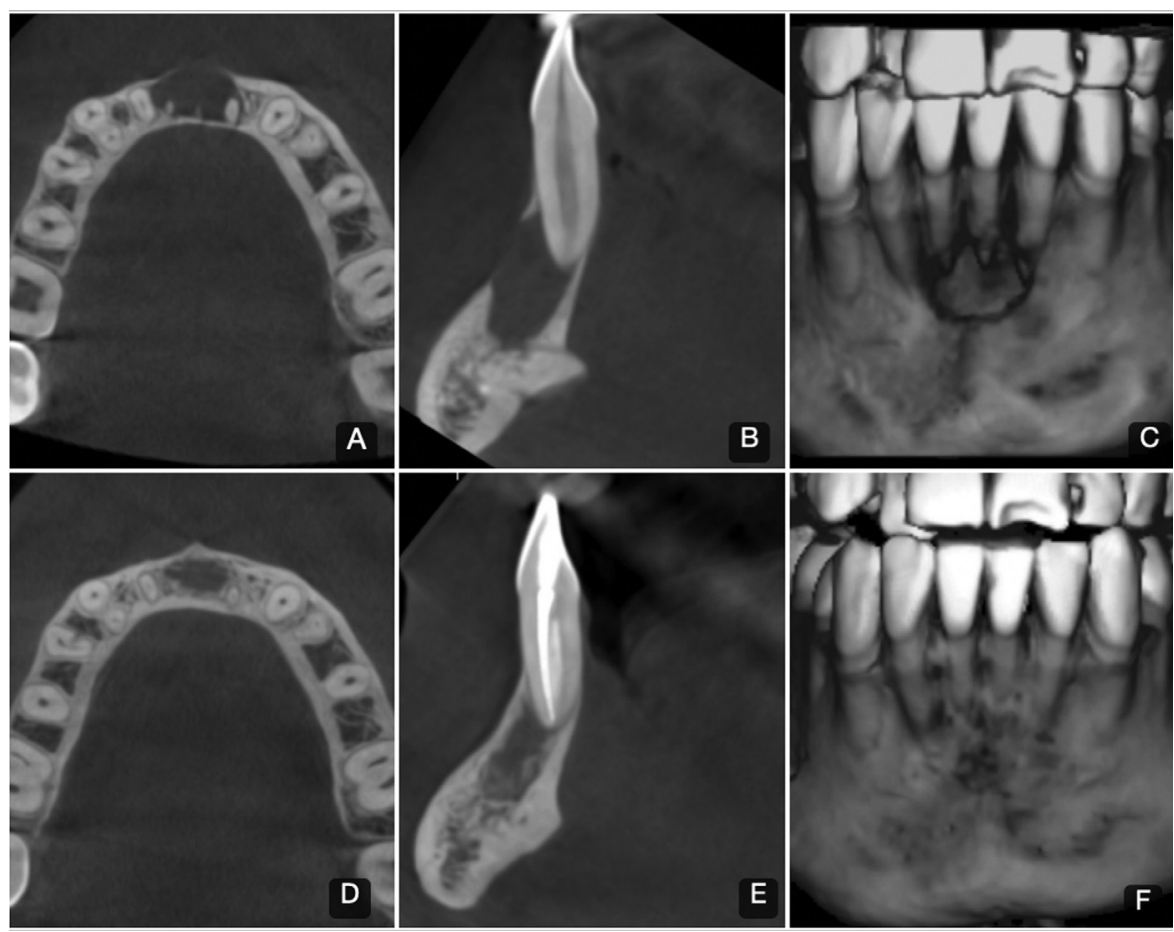

Fig. 1: CBCT images of Clinical Case \#1. A-B-C; Axial and sagittal view and three-dimensional reconstruction of the pre-treatment state. D-E-F; Axial and sagittal view and three-dimensional reconstruction of the post-treatment state after 6 months. Considerable diminution of the mesio-distal width can be observed, with peripheral areas of bone neoformation, recovery of lingual plate thickness and complete closure of the vestibular fenestration. 

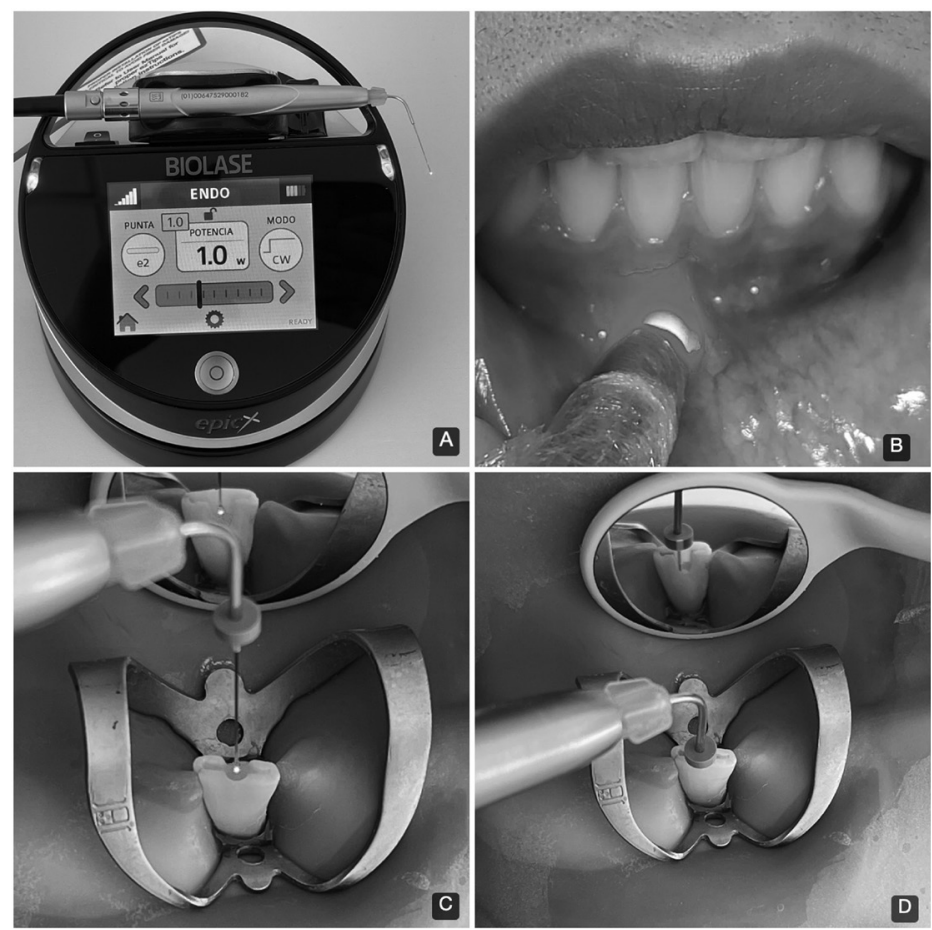

Fig. 2: A; 940-nm diode laser unit used in endodontic disinfection and PBMT in the two cases reported. B; Clinical image of application of the PBMT protocol. C-D; Intracanal application of diode laser.

$\mathrm{cm}^{2}$, power $0.1 \mathrm{~W}$, for $40 \mathrm{sec}$ per point, continuous mode, distributed in 4 points, using a total energy $16 \mathrm{~J}$ in an area of $2.6 \mathrm{~cm}^{2}$, with an energy density $6 \mathrm{~J} / \mathrm{cm}^{2}$ (Fig. 2). Surgical handpiece without tip was used for extraoral therapy..

The second session was carried out two weeks later. The same protocol of chemomechanical preparation (CMP) and laser disinfection were applied as described above. Root canal obturation was performed by the hydraulic method with bioceramic cement (Bioroot, Septodont, France). At the end of the first and second session, the same PBMT protocol was applied as described above. During the first week after completion of the endodontic treatment, one PBMT session was applied, followed by two in the second week and one each in the third and fourth weeks, making a total of 7 applications, all using the same protocol as described above. The defect volume and bone density were measured on CBCT images, initially and after 6 months post treatment (Fig. 1).

Case report 2. Female patient, 22 years old, with no relevant medical history, referred for assessment of apical radiolucency in teeth 3.2-3.1-4.1-4.2. Panoramic and pericapical X-ray examination showed a bilobulate osteolytic lesion, with mesio-distal width of $12.7 \mathrm{~mm}$, in the area of teeth 3.1 and 4.1, with expansion and fenestration of the vestibular cortical plate at tooth 4.1 (Fig. 3). Clinical examination showed soft tissues normal, percussion test and sensitivity thermal tests (ethyl chloride and guta-percha) negative. The endodontic diagno- sis was pulp necrosis/AAP. Endodontic treatment of tooth 4.1 (single root canal) was applied in 2 sessions. The protocol used for CMP, disinfection of the root canal by DL and PBMT (7 applications in total) and root canal obturation was the same as described above for case \#1. The defect volume and bone density were measured on CBCT images, initially and after 6 months post treatment (Fig. 3). The images were obtained on a Promax 3D CBCT unit (Planmeca, Helsinki, Finland), using 90 $\mathrm{kV}$ and $12 \mathrm{~mA}$; FOV $8 \times 8 \mathrm{~cm}$, voxel size $0.15 \mathrm{~mm}$, and analyzed with Romexis 4.5.1.R software (Planmeca, Helsinki, Finland).

Discussion

The antimicrobial effect of DL is based on a direct photothermal effect on the pigmented membranes of the bacteria. DL at wavelengths of 810,940 and $980 \mathrm{~nm}$ are hardly absorbed by water, allowing the light to interact with microorganisms located deep inside the dentin tubules. Conventional irrigation and ultrasonic activation are current techniques that present limitations in the disinfection of difficult-to-access areas, so the application of LLLT in endodontics seems to be a promising clinical alternative. Recently, Schulte-Lünzum et al. (8), using 940-nm DL with parameters recommended for endodontics (1-1.5W), observed a strong bactericidal effect against Enterococcus faecalis (83 to $99.99 \%$ ), even at a depth of $1000 \mu \mathrm{m}$, and with no adverse thermal effects. It should be noted that the thermal effect generated by DL can cause various complications inside the RCS if 


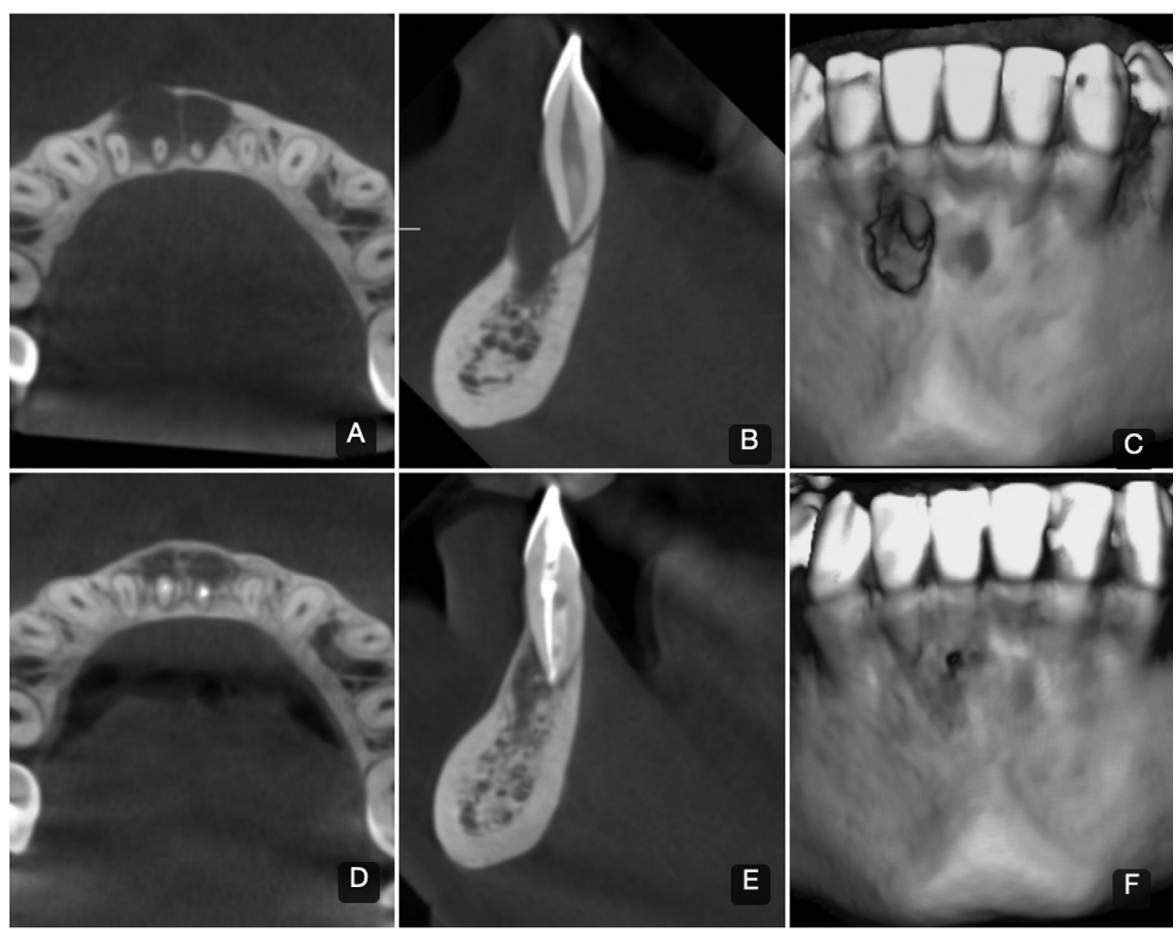

Fig. 3: CBCT images of Clinical Case \#2. A-B-C; Axial and sagittal view and three-dimensional reconstruction of the pre-treatment state. D-E-F; Axial and sagittal view and three-dimensional reconstruction of the post-treatment state after 6 months. A bony partition can be seen between the two hypodense focuses, with peripheral areas of bone neoformation and almost complete closure of the vestibular fenestration.

the parameters are not correctly regulated, such as root resorption and a change in dentin morphology (9). DL emits the energy in continuous wave (CW). However, $\mathrm{CW}$ in the root canal at higher power than $1.5 \mathrm{~W}$ is not advisable because of the heat damage that it may cause. It has been reported that if the laser tip comes into contact with the dentin wall during irradiation, it may cause heat damage, e.g. hot spots, fusion and cracks (9). For this reason, in the cases presented here we used a power of 1.0 W for disinfection of the RCS, based on endodontic protocols published previously (10). It has also been reported that the antimicrobial effectiveness of $\mathrm{NaOCl}$ is improved with 940-nm laser irradiation (10). It should therefore be noted that laser application is a complement to conventional CMP and not a substitute.

Postoperative pain is a frequent problem in endodontics. In the two cases reported here, the patients reported slight discomfort on the first day after treatment which diminished significantly on the second and third days. These observations agree with a recent study, in which postoperative pain diminished significantly on the third day after application of 940-nm DL in re-treatments (11). PBMT uses low-intensity energy to induce cell proliferation and improve the differentiation of stem cells. Red or near-infrared light wavelengths are those most commonly used in PBMT (600-1100 nm) (12). It has been shown that the use of a fluence in the range of 0.04-50
$\mathrm{J} / \mathrm{cm}^{2}$ is appropriate for generating optimum biological response (13). In the cases presented here, $6 \mathrm{~J} / \mathrm{cm}^{2}$ were applied, creating a biostimulating effect in both cases. It was shown that PBMT in a low energy density range accelerated repair in both cases, with bone neoformation, recovery of lingual cortical plate thickness and closure of the vestibular fenestration after 6 months. The explanation is that low-intensity energy stimulates release of the growth factors involved in the formation of epithelial cells, fibroblasts, collagen and vascular proliferation, as well as accelerating the synthesis of bone matrix due to increased vascularisation and reduced inflammatory response, with a significant increase of osteocytes in the irradiated bone (14). PBMT also appears to be a useful coadjuvant in the bone repair process, especially when associated with the use of bioceramics, since both accelerate the formation of bone tissue, promote the proliferation/maturation of osteoblast cells and accelerate bone regeneration (5).

A study using digital periapical radiography showed the effectiveness of LLLT repair through bone formation in cystic defects following cyst enucleation. After three months, the LLLT group presented a significantly greater increase in bone density than the control group (6). Metin et al. (7) evaluated the effect of LLLT on the cicatrization of hard and soft tissues after endodontic surgery, using CBCT. The authors observed significant- 
ly better results in terms of bone density, volume/area of the defect, and periapical index in the third month after the operation compared to the control group.

Nevertheless, not all reports have endorsed the use of LLLT in regenerative processes. Jakse et al. (15) found no positive effect of LLLT using DL at $680-\mathrm{nm}(75 \mathrm{~mW})$ in a trial of bone regeneration and osseointegration of dental implants. These observations disagree with the results that we obtained in the two cases reported here. One possible explanation for the failure of the treatment was the laser application protocol, with only three sessions during the first week postoperative, low energy density (3-4 J/cm2) and the limited penetration of the wavelength used (680-nm); this dose is nowconsidered low for bone regeneration treatments. In contrast, the protocol used by us included a longer period of PBMT application after completion of the endodontic treatment, which may explain the differences between both studies.

\section{Conclusions}

Based on the resolution of the cases, we can infer that the 940-nm DL effect could help accelerate the healing of the tissues affected by the AP. It should be taken into account that the application of LLLT is a complement to conventional endodontic therapy and not a replacement. To strengthen the evidence, further radomised clinical trial studies must be developed.

\section{References}

1. Segura-Egea JJ, Martín-González J, Cabanillas-Balsera D, Fouad AF, Velasco-Ortega E, López-López J. Association between diabetes and the prevalence of radiolucent periapical lesions in root-filled teeth: systematic review and meta-analysis. Clin Oral Investig. 2016;20:1133-41.

2. Jakovljevic A, Nikolic N, Jacimovic J, Pavlovic O, Milicic B, Beljic-Ivanovic K, et al. Prevalence of Apical Periodontitis and Conventional Nonsurgical Root Canal Treatment in General Adult Population: An Updated Systematic Review and Meta-analysis of Cross-sectional Studies Published between 2012 and 2020. J Endod. 2020;46:13711386.

3. Laukkanen E, Vehkalahti MM, Kotiranta AK. Impact of type of tooth on outcome of non-surgical root canal treatment. Clin Oral Invest. 2019;23:4011-4018.

4. Masilionyte M, Gutknecht N. Outcome of 940-nm diode laser-assisted endodontic treatment of teeth with apical periodontitis: a retrospective study of clinical cases. Laser Dent Sci. 2018;2:169-179.

5. Macedo AAP, Santos TD, Cunha JLS, de Souza Matos F, de Albuquerque Júnior RLC, Ribeiro M AG. Effect of laser photobiomodulation associated with a bioceramic cement on the repair of bone tissue in the femur of rats. J Photochem Photobiol B. 2020;205:111813.

6. Zaky A, El Shenawy H, Harhsh T, Shalash M, Awad N. Can low level laser therapy benefit bone regeneration in localized maxillary cystic defects?- a prospective randomized control trial. Open Access Maced J Med Sci. 2016;4:720-725.

7. Metin R, Tatli U, Evlice B. Effects of low-level laser therapy on soft and hard tissue healing after endodontic surgery. Lasers Med Sci. 2018;33:1699-1706.

8. Schulte-Lünzum R, Gutknecht N, Conrads G, Franzen R. The Impact of a $940 \mathrm{~nm}$ Diode Laser with Radial Firing Tip and Bare End Fiber Tip on Enterococcus faecalis in the Root Canal Wall Dentin of Bovine Teeth: An In Vitro Study. Photomed Laser Surg. 2017;35:357-363.
9. Asnaashari M, Mojahedi SM, Asadi Z, Azari-Marhabi S, Maleki A. A comparison of the antibacterial activity of the two methods of photodynamic therapy (using diode laser $810 \mathrm{~nm}$ and LED lamp 630 $\mathrm{nm}$ ) against Enterococcus faecalis in extracted human anterior teeth, Photodiagnosis Photodyn Ther. 2016;13: 233-237.

10. Tilakchand M, Singh NN, Yeli MM, Naik BD. Evaluation of the antibacterial efficacy of ezlase diode laser on the infected root canal system: An in vivo study. J Conserv Dent. 2018;21:306-10.

11. Genc Sen O, Kaya M. Effect of Root Canal Disinfection with a Diode Laser on Postoperative Pain After Endodontic Retreatment. Photobiomodul Photomed Laser Surg. 2019;37:85-90.

12. Hamblin MR. Photobiomodulation for traumatic brain injury and stroke. J Neurosci Res. 2018;96:731-743.

13. AlGhamdi KM, Kumar A, Moussa NA. Low-level laser therapy: A useful technique for enhancing the proliferation of various cultured cells. Lasers Med Sci. 2012;27:237-249.

14. Escudero JSB, Perez MGB, de Oliveira Rosso MP, Buchaim DV, Pomini KT, Campos LMG, et al. Photobiomodulation therapy (PBMT) in bone repair: A systematic review. Injury. 2019;50:1853-1867.

15. Jakse N, Payer M, Tangl S, Berghold A, Kirmeier R, Lorenzoni $\mathrm{M}$. Influence of low-level laser treatment on bone regeneration and osseointegration of dental implants following sinus augmentation. An experimental study on sheep. Clin Oral Implants Res. 2007;18:517-24.

Acknowledgements

Article financed in part by the Research Directorate of Universidad de La Frontera.

\section{Author Contributions}

All authors contributed to this work and read and approved the final version of the manuscript.

\section{Informed consent}

Informed consent was obtained from the patients for the publication of this report.

\section{Conflict of interest}

The authors declare that they have no conflicts of interest related to this study. 